\title{
Quantification of the Beneficial Effects of Compression Stockings on Symptoms of Exercise and Orthostatic Intolerance in Chronic Fatigue Syndrome/Myalgic Encephalomyelitis Patients
}

\author{
C. (Linda) M. C. van Campen ${ }^{1 *}$, Freek W. A. Verheugt ${ }^{2}$, Frans C. Visser ${ }^{1}$ \\ ${ }^{1}$ Stichting Cardiozorg, Hoofddorp, Netherlands \\ ${ }^{2}$ Radboud UMC, Department of Cardiology, Nijmegen, Netherlands \\ Email: *info@stichtingcardiozorg.nl
}

How to cite this paper: van Campen, C. (L) M.C., Verheugt, F.W.A. and Visser, F.C. (2018) Quantification of the Beneficial Effects of Compression Stockings on Symptoms of Exercise and Orthostatic Intolerance in Chronic Fatigue Syndrome/Myalgic Encephalomyelitis Patients. International Journal of Clinical Medicine, 9, 367-376.

https://doi.org/10.4236/ijcm.2018.95032

Received: March 22, 2018

Accepted: May 6, 2018

Published: May 9, 2018

Copyright $\odot 2018$ by authors and Scientific Research Publishing Inc. This work is licensed under the Creative Commons Attribution International License (CC BY 4.0).

http://creativecommons.org/licenses/by/4.0/

\section{(c) (i) Open Access}

\begin{abstract}
Chronic fatigue syndrome and myalgic encephalomyelitis (CFS/ME) are, amongst others, characterized by exercise intolerance, pain, post exertional malaise and orthostatic intolerance. It has been shown in venous disease and sport participation that compression stockings may improve exercise performance and reduce post exercise muscle soreness. Moreover, its use is advocated in orthostatic hypotension. Therefore, it was hypothesized that compression stockings may reduce symptomatology in CFS/ME patients. Methods: 100 patients used compression stockings class II for minimally 3 weeks and thereafter filled in a questionnaire, based on the Rand 36 physical activity questions $(\mathrm{n}=9)$, whether compression stockings changed perceived symptoms or not. Moreover, 7 questions referring to prolonged standing and sitting, to recovery post exercise, muscle pain during or immediately post exercise, and to dizziness/light-headedness during or immediately post exercise, while standing and during prolonged sitting were added. Questions were scored as 1: able to perform activity much less while wearing the stockings, 2 : perform activity somewhat less, 3: no perceived change in activity, 4: perform activity slightly better, 5 : able to perform activity much better while wearing the stockings. Results: In patients able to answer the question, all mean scores per activity were significantly higher than 3 , being no perceived change in activity while wearing the stockings. Subgroup analysis showed that patients with orthostatic intolerance reported higher effects than patients without orthostatic intolerance. Conclusion: This pilot study suggests that compression
\end{abstract}


stockings may be useful to reduce symptomatology of physical activities in CFS/ME patients, especially in patients with orthostatic intolerance. Larger prospective studies with hard endpoints are warranted.

\section{Keywords}

Chronic Fatigue Syndrome, Myalgic Encephalomyelitis, Compression Stockings, Exercise Tolerance, Orthostatic Intolerance

\section{Introduction}

The terms chronic fatigue syndrome and myalgic encephalomyelitis (CFS/ME) describe a complex physical illness characterized by debilitating fatigue, post-exertional malaise (PEM), orthostatic intolerance, pain, cognitive problems, sleep dysfunction and an array of other immune, neurological and autonomic symptoms. Rest and sleep produce only modest relief of fatigue/PEM and the other symptoms. As in other diseases, the disease severity ranges from mild to severe. In the most severe cases, the patient is bedridden all of the time [1].

Signs and symptoms have been described in a recent extensive review of literature by the American Institute of Medicine (IoM) and in other scientific journals [2] [3] [4] [5]. Although a host of abnormalities have been identified in $\mathrm{CFS} / \mathrm{ME}$, and in almost any organ, the exact pathophysiology remains at present unclear [2] [6].

One of the important symptoms is orthostatic intolerance. Orthostatic intolerance is defined as a clinical condition in which symptoms worsen upon assuming and maintaining upright posture and are ameliorated (although not necessarily abolished) by recumbency. This symptom is not part of the CFS criteria [7] and is one of many symptoms in the ME criterion of energy production/transportation impairments [1]. In contrast, orthostatic intolerance was highlighted in the IoM report and was added as a criterion for the diagnosis [5].

Although the incidence of orthostatic intolerance abnormalities (postural orthostatic tachycardia and different forms of orthostatic hypotension) in CFS/ME patients has been extensively described [8]-[17], data on treatment are limited. Mainly fluid/sodium intake, exercise training and counter manoeuvre advices have been described, as well as the use of fludrocortisone [18] [19] [20], midodrine [21], pyridostigmine [22], desmopressin [23] and beta-blockers in CFS/ME patients with postural orthostatic tachycardia syndrome (POTS) [24].

In the recent American syncope guidelines, the use of compression garments was advocated in patients with orthostatic hypotension [25]. The use of compression garment was shown to reduce orthostatic intolerance symptoms in these patients [26]. Moreover, compression stockings are widely used in sport participation [27] and may improve exercise tolerance in venous disease [28]. Therefore, it was hypothesized that treatment of compression stockings can be advantageous to the standard supportive measures in patients with CFS/ME. As 
this has not been described before, we performed a pilot study, investigating the effects of sport compression stockings on exercise tolerance, pain and dizziness/light-headedness in consecutive CFS/ME patients, irrespective of orthostatic intolerance complaints.

\section{Patients and Methods}

A total of 134 consecutive patients with CFS/ME were invited to participate. The diagnosis of CFS was based on the criteria of Fukuda et al. [7], the diagnosis of ME based on the ICC criteria [1]. Disease severity was scored according to the ICC criteria [1]. Based on history taking, the presence or absence of orthostatic intolerance symptoms was noted. All patients underwent tilt table testing and hemodynamic results were classified as normal heart rate and blood pressure response, POTS, classic and delayed hypotension.

Three patients did not give informed consent for the start of the study, 5 were excluded because they refused to wear the stockings, 6 were excluded because they did not complete the questionnaires, 6 were excluded because they had too many complaints while putting on the stockings. Eight were excluded because they wore the stockings shorter than 3 weeks. Finally 9 were excluded because of incomplete questionnaires at the time of analysis, leading to a total of 100patients.All patients gave written informed consent and the study was approved by the METC of the MC Slotervaart.

Participating patients were instructed to use sport compression stockings (CEP running 2.0) during several weeks on several days of the week (at least 3 weeks). No specific instructions for the use during specific activities were given, except for not wearing the stockings at night. At the end of the trial period, a Rand 36 questionnaire was filled-in [29] [30]. From the Rand 36 the scores of physical functioning, of energy/fatigue and of pain were noted.

In the second questionnaire patients were asked whether they perceived a change of the physical activity while using the compression stockings. The same physical activity characteristics of the Rand 36 questionnaire were used (question 3 - 7, 9 - 12: vigorous activities, moderate activities, lifting/carrying groceries, climbing several stairs, climbing one stair, walking more than $1 \mathrm{~km}$, walking half a $\mathrm{km}$, walking $100 \mathrm{~m}$ and bathing/dressing). Each physical activity question had a 6 item scale: able to perform activity much less while wearing the stockings (score 1), able to perform activity somewhat less while wearing (score 2), no perceived change in activity while wearing (score 3), able to perform activity slightly better while wearing (score 4), and able to perform activity much better while wearing the stockings (score 5), cannot answer the question (score 6). In addition, using the same 6 item scale, patients were asked for a perceived change during prolonged standing, during prolonged sitting, in recovery $1 \mathrm{hr}$ post exercise, for muscle pain during or immediately post exercise, in dizziness/light-headedness during or immediately post exercise, in dizziness/light-headedness while standing and in dizziness/light-headedness during prolonged sitting. 
Data analysis: the answers to the question able to perform the physical activity much less and somewhat less while wearing the compression stockings were combined to worse performance while wearing, and the performed physical activities slightly better and much better while wearing the stockings were combined to improved activity. This led to a 3 point scale: worse performance, neutral effect and improved performance of activity while wearing the compression stockings. The relative contribution of the 3 point scales were calculated, excluding the number of patients who could not answer the question.

\section{Statistical Analysis}

Data were analyzed using SPSS 21 (IBM). The data are presented as the mean $+/-$ SD. A p value < 0.05 was considered significantly different. Continuous data were compared using Student's $t$ test. Nominal data were compared using the Chi-square test or the Fisher exact test. A p value $<0.05$ was considered significantly different. Graphs were constructed using Graphpad Prism version 6.00, Graphpad software, La Jola California USA.

\section{Results}

Table 1 shows the baseline characteristics. All had CFS/ME according to international criteria. The majority were females, disease duration was $13 \pm 9$ years, most patients had mild severity of the disease. Physical functioning, fatigue and pain scores were all low, indicative of substantial disability. Ninety-one percent had a clinical suspicion of orthostatic intolerance, while $86 \%$ had a normal tilt table test.

Figure 1 shows the percentage of patients who perceived improved, neutral and worse performance of activities while wearing the stockings. The mean data per activity were all significantly different from neutral. The percentage of patients reporting improved activity varied between 26\% (improved dressing activity) and $73 \%$ (improved walking for $0.5 \mathrm{~km}$ ).

Table 1. Baseline characteristics of patients.

\begin{tabular}{cc}
\hline Male/female & $14 / 86$ \\
Age in years (mean \pm SD) & $44 \pm 11$ \\
Length in cm (mean \pm SD) & $173 \pm 9$ \\
Weight in $\mathrm{kg}($ mean \pm SD) & $72 \pm 15$ \\
BMI in kg/m ${ }^{2}$ (mean \pm SD) & $24.2 \pm 4.6$ \\
CFS/ME duration in years (mean \pm SD) & $13 \pm 9$ \\
ME severity 1/2/3 & $52 / 38 / 10$ \\
Rand 36 score: physical functioning (mean \pm SD) & $34 \pm 20$ \\
Rand 36 score: energy/fatigue (mean \pm SD) & $44 \pm 10$ \\
Rand 36 score: pain (mean \pm SD) & $46 \pm 24$ \\
Orthostatic intolerance complaints yes/no & $91 / 9$ \\
Tilt table results: normal/POTS/delayed OH & $86 / 5 / 3$ \\
\hline
\end{tabular}




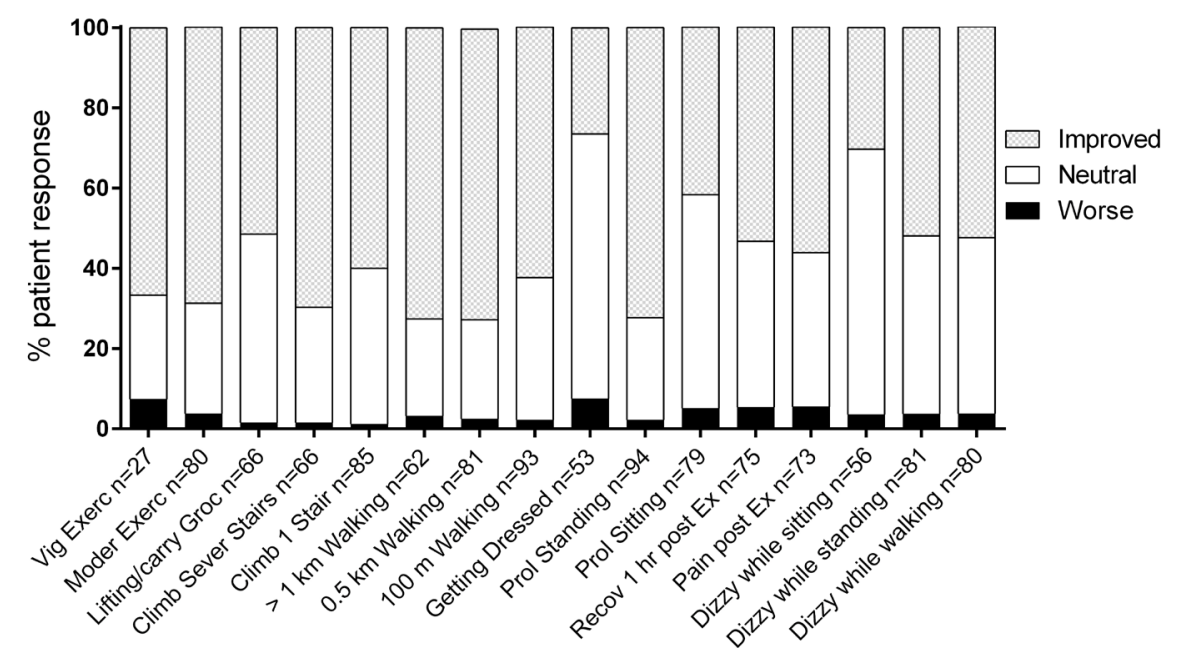

Figure 1. The distribution of perceived effects of activities while wearing compression stockings. The numbers in the $\mathrm{X}$ axis titles refer to number of patients able to answer the questions. VigExerc: vigorous exercise; ModerExerc: moderate exercise; Lifting/carry Groc: Lifting or carrying groceries; Climb Sever Stairs: climbing several stairs; Climb 1 Stair: climbing of 1 stair; Prol Standing: prolonged standing; Prol Sitting: prolonged sitting; Recov $1 \mathrm{hr}$ post Ex: recovery $1 \mathrm{hr}$ post exercise; Pain post Ex: muscle pain during or post exercise: Dizzy while sitting: dizziness/lightheadedness while sitting; Dizzy while standing: dizziness/lightheadedness while standing; Dizzy while walking: dizziness/lightheadedness while walking.

In a further analysis, patients were divided in 2 groups: with $(\mathrm{n}=91)$ and without $(n=9)$ orthostatic intolerance complaints. No differences between the 2 groups were found in gender distribution, age, CFS/ME duration, and disease severity, Rand 36 scores for physical functioning, energy/fatigue and pain, and in the hemodynamic abnormality distribution. Table 2 shows the scores for the perceived change in activities while wearing the stockings of patients with and without orthostatic intolerance orthostatic intolerance complaints. In patients without orthostatic intolerance complaints, none of the scores of activities were significantly different from neutral (a score of 3). In contrast, all mean scores of activities were significantly higher than neutral in patients with orthostatic intolerance complaints. A comparison of patients with and without orthostatic intolerance complaints showed that the differences were confined to various physical activities, whereas the effect on pain, dizziness/light headedness and functional recovery after exercise were not significantly different.

\section{Discussion}

Compression stockings are widely in various venous and lymphatic diseases [28], although a formal guideline is lacking. One of the conclusions of the authors of this consensus statement was that the use resulted in a significant trend towards an improvement in the ability to perform physical exercise. Furthermore, in the recent American syncope guidelines the use of compression garments were advocated in patients with orthostatic hypotension [25], where the 
Table 2. Mean scores of activity questions in patients with and without orthostatic intolerance complaints.

\begin{tabular}{cccc}
\hline & OI - Patients & OI + Patients & P value between groups \\
\hline Number & 9 & 91 & \\
Vigorous Exercise & $2.5 \pm 0.7$ & $3.9 \pm 0.8^{\# \# \# \#}$ & 0.05 \\
Moderate Exercise & $3.3 \pm 1.0$ & $3.9 \pm 0.7^{\# \# \# \#}$ & 0.05 \\
Lifting/carrying groceries & $3.3 \pm 1.0$ & $3.7 \pm 0.7^{\# \# \# \#}$ & $\mathrm{~ns}$ \\
Climb Several stairs & $3.0 \pm 0.7$ & $4.0 \pm 0.7^{\# \# \# \#}$ & 0.005 \\
Climb One stair & $3.1 \pm 0.7$ & $3.9 \pm 0.8^{\# \# \#}$ & 0.005 \\
$>1$ km Walking & $3.0 \pm 0.7$ & $4.1 \pm 0.8^{\# \# \#}$ & 0.005 \\
0.5 km Walking & $3.1 \pm 0.6$ & $3.9 \pm 0.7^{\# \# \# \#}$ & 0.05 \\
100 m Walking & $3.3 \pm 0.8$ & $4.0 \pm 0.7^{\# \# \# \#}$ & $\mathrm{~ns}$ \\
Dressing & $3.2 \pm 0.5$ & $3.2 \pm 0.6^{\#}$ & $\mathrm{~ns}$ \\
Prolonged Standing & $3.3 \pm 0.7$ & $4.0 \pm 0.7^{\# \# \# \#}$ & 0.05 \\
Prolonged Sitting & $3.1 \pm 0.7$ & $3.5 \pm 0.7^{\# \# \# \#}$ & $\mathrm{~ns}$ \\
Recovery after 1 hr exercise & $3.4 \pm 0.5$ & $3.6 \pm 0.8^{\# \# \# \#}$ & $\mathrm{~ns}$ \\
Muscle pain during/after exercise & $3.3 \pm 0.5$ & $3.8 \pm 0.9^{\# \# \# \#}$ & $\mathrm{~ns}$ \\
Dizziness while sitting & $3.0 \pm 0.8$ & $3.4 \pm 0.7^{\# \# \# \#}$ & $\mathrm{~ns}$ \\
Dizziness while standing & $3.0 \pm 0.6$ & $3.6 \pm 0.8^{\# \# \#}$ & $\mathrm{~ns}$ \\
Dizziness during exercise & $3.1 \pm 0.7$ & $3.6 \pm 0.8^{\# \# \# \#}$ & $\mathrm{~ns}$ \\
\hline
\end{tabular}

": $\mathrm{p}<0.05$ (higher than neutral effect $=$ score 3 ); ${ }^{\# \# \# \#: ~} \mathrm{p}<0.001$ (higher than neutral effect $=$ score 3 ).

use of compression garment was shown to reduce orthostatic intolerance symptoms [26]. Finally, although there are conflicting results regarding the effects of wearing compression garments during exercise, there is a trend towards a beneficial effect of compression garments worn during recovery [27]. As exercise intolerance, post exertional malaise and orthostatic intolerance are main complaints in CFS/ME patients, and as scientific data are missing in this patient group, a pilot study was performed to assess its use in daily practice.

This study of 100 consecutive CFS/ME patients suggests that the use of compression stockings resulted in an improved perceived activity: all mean activity scores were higher than neutral, being a score of 3 . As expected, the percentage of patients reporting improvement varied widely, with a range between $26 \%$ (improved dressing activity) and 73\% (improved walking for $0.5 \mathrm{~km}$ ): (see Figure 1).

A further sub-analysis showed that the main effects were present in patients with orthostatic intolerance complaints (see Table 2): for all reported activities the mean scores were significantly higher than a score of 3 (neutral effect), whereas in patients without orthostatic intolerance the mean reported effects on activity were not significantly different from a neutral effect. This suggests that the beneficial effects are confined to those patients with orthostatic intolerance. However, the number of patients without orthostatic intolerance was low and further larger prospective studies with solid endpoints are needed to address this 
question.

The mechanisms how compression stockings exert their beneficial effects are complex. Studies in other pathologies and during exercise show increased venous flow [31], improved lymphatic drainage [32], improved tissue oxygenation [33], reduction of pro-inflammatory cytokines [34], improved arterial flow post exercise [35], and improved endothelial function [36] while wearing compression stockings. The mechanism of the potential beneficial effects of compression stockings has not been studied in ME/CFS patients. However, in one study a counter pressure of $45 \mathrm{mmHg}$ (using military anti-shock trousers) during prolonged standing in ME/CFS patients normalized the excessive heart rate increase and the excessive decrease in blood pressures [37]. This observation strengthens the potential use of compression stockings.

In the present study class II stockings were used. In a recent study the degree of compression on exercise performance and recovery was reviewed [27]. The authors concluded that there was no apparent relation between the effects of compression garments worn during or after exercise and the pressures applied, since beneficial effects were obtained with both low and high pressures [27]. Whether high pressure stockings in CFS/ME patients are more beneficial than low pressure stockings need to be determined.

Limitations: firstly, the use of questions, although similar to the Rand 36 physical activity questions, was not validated both internally and externally. Also, the questionnaire was filled-in only after the use of the compression stockings. However, the sole purpose of this pilot study was to determine whether there was any beneficial effect. Giving the results, a prospective study with objective outcome measures and predefined CFS/ME subgroups is justified, as are studies elucidating the underlying mechanisms of the effects of compression stockings.

\section{Conclusion}

So far, the use of compression stockings in CFS/ME patients has not been studied before. This pilot study suggests that wearing compression stockings during daily life of CFS/ME patients are useful to reduce symptomatology of physical activities. The data also suggest that the subgroup of patients with orthostatic intolerance especially benefits from the use of stockings. Larger, prospective studies are warranted.

\section{Funding}

This research received no specific grant from any funding agency in the public, commercial, or not-for-profit sectors.

\section{References}

[1] Carruthers, B.M., et al. (2011) Myalgic Encephalomyelitis: International Consensus Criteria. Journal of Internal Medicine, 270, 327-338. 
https://doi.org/10.1111/j.1365-2796.2011.02428.x

[2] Clayton, E.W. (2015) Beyond Myalgic Encephalomyelitis/Chronic Fatigue Syndrome: An IOM Report on Redefining an Illness. JAMA, 313, 1101-1102. https://doi.org/10.1001/jama.2015.1346

[3] Jason, L.A., et al. (2015) Problems in Defining Post-Exertional Malaise. Journal of Prevention \& Intervention in the Community, 43, 20-31. https://doi.org/10.1080/10852352.2014.973239

[4] Loy, B.D., O’Connor, P.J. and Dishman, R.K. (2016) Effect of Acute Exercise on Fatigue in People with ME/CFS/SEID: A Meta-Analysis. Medicine \& Science in Sports \& Exercise, 48, 2003-2012. https://doi.org/10.1249/MSS.0000000000000990

[5] IOM. (2015) Beyond Mayalgic Encephalomyelitis/Chronic Fatigue Syndrome: Redefining an Illness. The National Academies Press, Washington DC.

https://doi.org/10.1186/1741-7015-11-205

[6] Morris, G. and Maes, M. (2013) Myalgic Encephalomyelitis/Chronic Fatigue Syndrome and Encephalomyelitis Disseminata/Multiple Sclerosis Show Remarkable Levels of Similarity in Phenomenology and Neuroimmune Characteristics. BMC Medicine, 11, 205. https://doi.org/10.1186/1741-7015-11-205

[7] Fukuda, K., et al. (1994) The Chronic Fatigue Syndrome: A Comprehensive Approach to Its Definition and Study. International Chronic Fatigue Syndrome Study Group. Annals of Internal Medicine, 121, 953-959. https://doi.org/10.7326/0003-4819-121-12-199412150-00009

[8] Bou-Holaigah, I., et al. (1995) The Relationship between Neurally Mediated Hypotension and the Chronic Fatigue Syndrome. JAMA, 274, 961-967.

https://doi.org/10.1001/jama.1995.03530120053041

[9] De Lorenzo, F., Hargreaves, J. and Kakkar, V.V. (1997) Pathogenesis and Management of Delayed Orthostatic Hypotension in Patients with Chronic Fatigue Syndrome. Clinical Autonomic Research, 7, 185-190.

https://doi.org/10.1007/BF02267980

[10] Roerink, M.E., et al. (2017) Postural Orthostatic Tachycardia Is Not a Useful Diagnostic marker for Chronic Fatigue Syndrome. Journal of Internal Medicine, 281, 179-188. https://doi.org/10.1111/joim.12564

[11] Miwa, K. (2016) Variability of Postural Orthostatic Tachycardia in Patients with Myalgic Encephalomyelitis and Orthostatic Intolerance. Heart Vessels, 31, 1522-1528. https://doi.org/10.1007/s00380-015-0744-3

[12] Razumovsky, A.Y., et al. (2003) Cerebral and Systemic Hemodynamics Changes during Upright Tilt in Chronic Fatigue Syndrome. Journal of Neuroimaging, 13, 57-67. https://doi.org/10.1111/j.1552-6569.2003.tb00158.x

[13] Hoad, A., et al. (2008) Postural Orthostatic Tachycardia Syndrome Is an Under-Recognized Condition in Chronic Fatigue Syndrome. QJM, 101, 961-965. https://doi.org/10.1093/qjmed/hcn123

[14] Lewis, I., et al. (2013) Clinical Characteristics of a Novel Subgroup of Chronic Fatigue Syndrome Patients with Postural Orthostatic Tachycardia Syndrome. Journal of Internal Medicine, 273, 501-510. https://doi.org/10.1111/joim.12022

[15] Reynolds, G.K., et al. (2014) Comorbidity of Postural Orthostatic Tachycardia Syndrome and Chronic Fatigue Syndrome in an Australian Cohort. Journal of Internal Medicine, 275, 409-417. https://doi.org/10.1111/joim.12161

[16] LaManca, J.J., et al. (1999) Cardiovascular Response during Head-Up Tilt in Chronic Fatigue Syndrome. Clinical Physiology, 19, 111-120. https://doi.org/10.1046/j.1365-2281.1999.00154.x 
[17] Jones, J.F., et al. (2005) Orthostatic Instability in a Population-Based Study of Chronic Fatigue Syndrome. American Journal of Medicine, 118, 1415. https://doi.org/10.1016/j.amjmed.2005.06.013

[18] Blockmans, D., et al. (2003) Combination Therapy with Hydrocortisone and Fludrocortisone Does Not Improve Symptoms in Chronic Fatigue Syndrome: A Randomized, Placebo-Controlled, Double-Blind, Crossover Study. American Journal of Medicine, 114, 736-741. https://doi.org/10.1016/S0002-9343(03)00182-7

[19] Peterson, P.K., et al. (1998) A Preliminary Placebo-Controlled Crossover Trial of Fludrocortisone for Chronic Fatigue Syndrome. Archives of Internal Medicine, 158, 908-914. https://doi.org/10.1001/archinte.158.8.908

[20] Rowe, P.C., et al. (2001) Fludrocortisone Acetate to Treat Neurally Mediated Hypotension in Chronic Fatigue Syndrome: A Randomized Controlled Trial. JAMA, 285, 52-59. https://doi.org/10.1001/jama.285.1.52

[21] Naschitz, J., et al. (2004) Midodrine Treatment for Chronic Fatigue Syndrome. Postgraduate Medical Journal, 80, 230-232. https://doi.org/10.1136/pgmj.2003.011429

[22] Kawamura, Y., et al. (2003) Efficacy of a Half Dose of Oral Pyridostigmine in the Treatment of Chronic Fatigue Syndrome: Three Case Reports. Pathophysiology, 9, 189-194. https://doi.org/10.1016/S0928-4680(03)00007-5

[23] Miwa, K. (2017) Down-Regulation of Renin-Aldosterone and Antidiuretic Hormone Systems in Patients with Myalgic Encephalomyelitis/Chronic Fatigue Syndrome. Journal of Cardiology, 69, 684-688. https://doi.org/10.1016/j.jjcc.2016.06.003

[24] McDonald, C., et al. (2014) Postural Tachycardia Syndrome Is Associated with Significant Symptoms and Functional Impairment Predominantly Affecting Young Women: A UK Perspective. BMJ Open, 4, e004127.

https://doi.org/10.1136/bmjopen-2013-004127

[25] Shen, W.K., et al. (2017) 2017 ACC/AHA/HRS Guideline for the Evaluation and Management of Patients with Syncope: Executive Summary: A Report of the American College of Cardiology/American Heart Association Task Force on Clinical Practice Guidelines and the Heart Rhythm Society. Journal of the American College of Cardiology, 70, 620-663. https://doi.org/10.1016/j.jacc.2017.03.002

[26] Podoleanu, C., et al. (2006) Lower Limb and Abdominal Compression Bandages Prevent Progressive Orthostatic Hypotension in Elderly Persons: A Randomized Single-Blind Controlled Study. Journal of the American College of Cardiology, 48, 1425-1432. https://doi.org/10.1016/j.jacc.2006.06.052

[27] Beliard, S., et al. (2015) Compression Garments and Exercise: No Influence of Pressure Applied. Journal of Sports Science and Medicine, 14, 75-83.

[28] Rabe, E., et al. (2018) Indications for Medical Compression Stockings in Venous and Lymphatic Disorders: An Evidence-Based Consensus Statement. Phlebology, 33, 163-184. https://doi.org/10.1177/0268355516689631

[29] Murdock, K.W., et al. (2017) The Utility of Patient-Reported Outcome Measures among Patients with Myalgic Encephalomyelitis/Chronic Fatigue Syndrome. Quality of Life Research, 26, 913-921. https://doi.org/10.1007/s11136-016-1406-3

[30] Haywood, K.L., Staniszewska, S. and Chapman, S. (2012) Quality and Acceptability of Patient-Reported Outcome Measures Used in Chronic Fatigue Syndrome/Myalgic Encephalomyelitis (CFS/ME): A Systematic Review. Quality of Life Research, 21, 35-52. https://doi.org/10.1007/s11136-011-9921-8

[31] Motykie, G.D., et al. (1999) Evaluation of Therapeutic Compression Stockings in 
the Treatment of Chronic Venous Insufficiency. Dermatologic Surgery, 25, 116-120. https://doi.org/10.1046/j.1524-4725.1999.08095.x

[32] Moffatt, C. (2008) Variability of Pressure Provided by Sustained Compression. International Wound Journal, 5, 259-265. https://doi.org/10.1111/j.1742-481X.2008.00470.x

[33] Agu, O., Baker, D. and Seifalian, A.M. (2004) Effect of Graduated Compression Stockings on Limb Oxygenation and Venous Function during Exercise in Patients with Venous Insufficiency. Vascular, 12, 69-76.

https://doi.org/10.1258/rsmvasc.12.1.69

[34] Beidler, S.K., et al. (2009) Inflammatory Cytokine Levels in Chronic Venous Insufficiency Ulcer Tissue before and after Compression Therapy. Journal of Vascular Surgery, 49, 1013-1020. https://doi.org/10.1016/j.jvs.2008.11.049

[35] Mann, S., et al. (2016) The Effects of Compression Socks on Arterial Blood Flow and Arterial Reserves in Amateur Sportsmen. Development in Sports Science, 1, $1-9$.

[36] Okamoto, T., et al. (2013) Acute Effect of Brisk Walking with Graduated Compression Stockings on Vascular Endothelial Function and Oxidative Stress. Clinical Physiology and Functional Imaging, 33, 455-462. https://doi.org/10.1111/cpf.12052

[37] Streeten, D.H., Thomas, D. and Bell, D.S. (2000) The Roles of Orthostatic Hypotension, Orthostatic Tachycardia, and Subnormal Erythrocyte Volume in the Pathogenesis of the Chronic Fatigue Syndrome. The American Journal of the Medical Sciences, 320, 1-8. https://doi.org/10.1097/00000441-200007000-00001 